\title{
A NOTE ON SYSTEMS OF HOMOGENEOUS ALGEBRAIC EQUATIONS
}

\author{
RICHARD BRAUER
}

1. Introduction. Consider a system of algebraic equations

(1)

$$
\begin{gathered}
f_{1}\left(x_{1}, x_{2}, \cdots, x_{n}\right)=0, \\
f_{2}\left(x_{1}, x_{2}, \cdots, x_{n}\right)=0, \\
. . . . \cdot \cdot \\
f_{h}\left(x_{1}, x_{2}, \cdots, x_{n}\right)=0
\end{gathered}
$$

where $f_{i}$ is a homogeneous polynomial of degree $r_{i}$ with coefficients belonging to a given field $K$. We interpret $x_{1}, x_{2}, \cdots, x_{n}$ as homogeneous coordinates in an $(n-1)$-dimensional projective space. When $n>h$, the system (1) has non-trivial solutions $\left(x_{1}, x_{2}, \cdots, x_{n}\right)$ in an algebraically closed extension field of $K$, but there may not exist any such solutions in $K$ itself. It is, in general, extremely difficult to decide whether adjunction of irrationalities of a certain type to $K$ is sufficient to guarantee the existence of non-trivial solutions of (1) in the extended field. However, the situation is much simpler, when $n$ is very large, in the sense that $n$ lies above a certain expression depending on the number of equations $h$ and the degrees $r_{1}, r_{2}, \cdots, r_{h}$.

We shall show:

TheOREM A. For any system of $h$ positive degrees $r_{1}, r_{2}, \cdots, r_{h}$ there exists an integer $\Phi\left(r_{1}, r_{2}, \cdots, r_{h}\right)$ such that for $n \geqq \Phi\left(r_{1}, r_{2}, \cdots, r_{h}\right)$ the system (1) has a non-trivial solution in a soluble extension field $K_{1}$ of $K$. The field $K_{1}$ may be chosen such that its degree $N_{1}$ over $K$ lies below a value depending on $r_{1}, r_{2}, \cdots, r_{h}$ alone and that any prime factor of $N_{1}$ is at most equal to $\max \left(r_{1}, r_{2}, \cdots, r_{h}\right)$.

This Theorem A is evidently contained in the following theorem.

TheOREM B. For any system of positive integers $r_{1}, r_{2}, \cdots, r_{h}$ and any integer $m \geqq 0$, there exists an integer $\Phi\left(r_{1}, r_{2}, \cdots, r_{h} ; m\right)$ with the following property: For $n \geqq \Phi\left(r_{1}, \cdots, r_{h} ; m\right)$, there exists a soluble extension field $K_{2}$ of $K$ such that all points $\left(x_{1}, x_{2}, \cdots, x_{n}\right)$ of an m-dimensional linear manifold $L$, defined in $K_{2}$, satisfy the equations (1). Here $K_{2}$ may be chosen so that its degree $\mathrm{N}_{2}$ over $K$ lies below a bound depending on $r_{1}, r_{2}, \cdots, r_{h}$ and $m$ and that no prime factor of $N_{2}$ exceeds $\max \left(r_{1}, r_{2}, \cdots, r_{h}\right)$. 1945.

Presented to the Society, September 17, 1945; received by the editors July 17, 
At the same time, we shall prove the theorem:

Theorem C. Assume that the field $K$ has the following property:

$\left.{ }^{*}\right)$ For every integer $r>0$, there exists an integer $\Psi(r)$ such that for $n \geqq \Psi(r)$ every equation

$$
a_{1} x_{1}^{r}+a_{2} x_{2}^{r}+\cdots+a_{n} x_{n}^{r}=0
$$

with coefficients $a_{i}$ in $K$ has a non-trivial solution in $K$.

Then, for every system of positive degrees $r_{1}, r_{2}, \cdots, r_{h}$ and every integer $m \geqq 0$, there exists an expression $\Omega\left(r_{1}, r_{2}, \cdots, r_{h} ; m\right)$ with the following property: For $n \geqq \Omega\left(r_{1}, r_{2}, \cdots, r_{h} ; m\right)$, there exists an m-dimensional linear manifold $M$, defined in $K$, whose points satisfy the equations (1).

We shall prove Theorem $\mathrm{C}$ in $\S 2$. The changes necessary in order to obtain Theorem B are obvious. In $\S 3$, some applications are given. One of them is concerned with Hilbert's resolvent problem. We prove here a recent conjecture of B. Segre. ${ }^{1}$

2. Proof of Theorem C. 1. Assume that Theorem C is not true. We choose a system $r_{1}, r_{2}, \cdots, r_{h} ; m$ for which no $\Omega\left(r_{1}, \cdots, r_{h} ; m\right)$ exists. We select this system such that $\max \left(r_{1}, \cdots, r_{h}\right)=s$ has the smallest possible value, and that for fixed $s$ the number $h$ has the smallest possible value. If $r_{1}^{\prime}, r_{2}^{\prime}, \cdots, r_{h^{\prime}}^{\prime}$ is any system of positive integers and $m^{\prime}$ a non-negative integer, then $\Omega\left(r_{1}^{\prime}, r_{2}^{\prime}, \cdots, r_{h^{\prime}}^{\prime} ; m^{\prime}\right)$ exists, if either

$$
\max \left(r_{1}^{\prime}, r_{2}^{\prime}, \cdots, r_{h^{\prime}}^{\prime}\right)<s
$$

or if

$$
\max \left(r_{1}^{\prime}, r_{2}^{\prime}, \cdots, r_{h^{\prime}}^{\prime}\right)=s, \quad h^{\prime}<h .
$$

Assume first that $h>1$. We may assume that $r_{h}=s$. It follows that $\Omega\left(r_{1}, r_{2}, \cdots, r_{h-1} ; m\right)$ exists (cf. the conditions (3a) and (3b)) and also that $\Omega\left(s ; m^{\prime}-1\right)$ exists for any integer $m^{\prime}>0$. We set $m^{\prime}=\Omega\left(r_{1}, \cdots, r_{h-1} ; m\right)$. If $n \geqq \Omega\left(s ; m^{\prime}-1\right)$, the equation $f_{h}=0$ is satisfied by all points of an $\left(m^{\prime}-1\right)$-dimensional linear manifold $M_{1}$. If we restrict ourselves to points of $M_{1}$, we may express $x_{1}, \cdots, x_{n}$ linearly and homogeneously by $m^{\prime}$ parameters $y_{1}, \cdots, y_{m^{\prime}}$ with coefficients in $K$. Then $f_{i}\left(x_{1}, \cdots, x_{n}\right)$ becomes a homogeneous polynomial $g_{i}$ of $y_{1}, \cdots, y_{m^{\prime}}$. The degree of $g_{i}$ is $r_{i}$; the coefficients of $g_{i}$ belong to $K$. In particular, $g_{h}$ vanishes identically. In order to solve

${ }^{1}$ B. Segre, Ann. of Math. vol. 46 (1945) p. 287. Added September 10: In the meantime, I learned from Mr. Segre that he also found Theorem A from which the proof of the conjecture can be derived. 
(1), we have to solve

$$
g_{1}=0, g_{2}=0, \cdots, g_{h-1}=0 .
$$

Since $m^{\prime}=\Omega\left(r_{1}, \cdots, r_{h-1} ; m\right)$, the equations (4) will be satisfied by all points of an $m$-dimensional manifold $M_{2}$ of the $\left(y_{1}, \cdots, y_{m^{\prime}}\right)$-space. This then gives an $m$-dimensional linear manifold of the $\left(x_{1}, \cdots, x_{n}\right)$ space for which the equations (1) hold. But this shows that the expression $\Omega\left(r_{1}, \cdots, r_{h} ; m\right)$ exists; we may take

$$
\Omega\left(r_{1}, \cdots, r_{h} ; m\right)=\Omega\left(\max \left(r_{1}, \cdots, r_{h}\right) ; \Omega\left(r_{1}, \cdots, r_{h-1} ; m\right)-1\right) .
$$

Hence the case $h>1$ is impossible.

2. We now consider the case $h=1$. The system (1) consists of only one equation

$$
f\left(x_{1}, x_{2}, \cdots, x_{n}\right)=0
$$

of degree $r_{1}=s$.

From the way the number $s$ was chosen it follows that $\Omega(s ; m)$ does not exist while for every system $r_{1}^{\prime}, r_{2}^{\prime}, \cdots, r_{h^{\prime}}^{\prime}$ with $r_{1}^{\prime}<s$, $r_{2}^{\prime}<s, \cdots, r_{h^{\prime}}^{\prime}<s$ and all $m^{\prime}$ the existence of $\Omega\left(r_{1}^{\prime}, r_{2}^{\prime}, \cdots, r_{h^{\prime}}^{\prime} ; m^{\prime}\right)$ may be assumed.

We first discuss the case $m=0$. Denoting the point $\left(x_{1}, x_{2}, \cdots, x_{n}\right)$ by $\mathfrak{x}$, we write $f\left(x_{1}, x_{2}, \cdots, x_{n}\right)=f(\mathfrak{x})$.

If $\mathfrak{x}_{1}, \mathfrak{x}_{2}, \cdots, \mathfrak{x}_{n}$ are $n$ points whose coordinates are independent indeterminates and if $u_{1}, u_{2}, \cdots, u_{n}$ are $n$ further independent indeterminates, we may set

(5) $f\left(u_{1} \mathfrak{x}_{1}+u_{2} \mathfrak{x}_{2}+\cdots+u_{n} \mathfrak{x}_{n}\right)=\sum u_{1}^{\mu} u_{2}^{\nu} \cdots u_{n}^{\tau} f_{\mu \nu} \ldots_{\tau}\left(\mathfrak{x}_{1}, \mathfrak{x}_{2}, \cdots, \mathfrak{x}_{n}\right)$,

where the sum on the right side extends over all systems of $n$ nonnegative integers $(\mu, \nu, \cdots, \tau)$ with

$$
\mu+\nu+\cdots+\tau=s \text {. }
$$

The expressions $f_{\mu, \nu}, \ldots, \tau\left(\mathfrak{x}_{1}, \mathfrak{x}_{2}, \cdots, \mathfrak{x}_{n}\right)$ (the polar forms of $f$ ) are homogeneous polynomials in the coordinates of each $\mathfrak{x}_{i}$. As is easily seen, $f_{\mu, \nu}, \ldots, \tau\left(\mathfrak{x}_{1}, \mathfrak{x}_{2}, \cdots, \mathfrak{x}_{n}\right)$ is of degree $\mu$ in the coordinates of $\mathfrak{x}_{1}$, of degree $\nu$ in the coordinates of $\mathfrak{x}_{2}, \cdots$, of degree $\tau$ in the coordinates of $\mathfrak{x}_{n}$.

Let $a_{1} \neq 0$ be a fixed point. ${ }^{2}$ Choose $n-1$ points $e_{1}, e_{2}, \cdots, e_{n-1}$ which together with $\mathfrak{a}_{1}$ form a full linearly independent system, and set $\mathfrak{y}=y_{1} \mathrm{e}_{1}+y_{2} \mathrm{e}_{2}+\cdots+y_{n-1} \mathfrak{e}_{n-1}$ where the coefficients $y_{1}, y_{2}, \cdots, y_{n-1}$ are indeterminates.

Consider the system of equations

${ }^{2}$ We denote by $\mathrm{o}$ the row $(0,0, \cdots, 0)$ consisting of $n$ numbers 0 . 


$$
\begin{aligned}
& f_{\mathfrak{s}-1,1,0, \cdots, 0}\left(\mathfrak{a}_{1}, \mathfrak{y}, \mathfrak{D}, \cdots, \mathfrak{o}\right)=0, \\
& f_{s-2,2,0}, \cdots, 0\left(\mathfrak{a}_{1}, \mathfrak{y}, \mathfrak{D}, \cdots, \mathfrak{D}\right)=0, \\
& f_{1,8-1,0}, \cdots, 0\left(\mathfrak{a}_{1}, \mathfrak{y}, \mathfrak{b}, \cdots, \mathfrak{b}\right)=0 .
\end{aligned}
$$

These equations are homogeneous in $y_{1}, y_{2}, \cdots, y_{n-1}$; the degrees are $1,2, \cdots, s-1$ respectively.

From the remarks above it follows that the expression $\Omega(1,2, \cdots$, $s-1 ; 0)$ exists. Hence for sufficiently large $^{3} n$ the equations (6) will have a non-trivial solution. Let $\mathfrak{y}=\mathfrak{a}_{2}$ be the corresponding point $\mathfrak{y}$. Then $\mathfrak{a}_{1}$ and $\mathfrak{a}_{2}$ are linearly independent.

Let $\mathfrak{e}_{1}^{\prime}, \mathfrak{e}_{2}^{\prime}, \cdots, \mathfrak{e}_{n-2}^{\prime}$ be a system of points which together with $\mathfrak{a}_{1}$ and $\mathfrak{a}_{2}$ form a full linearly independent system and set

$$
z=z_{1} \mathrm{e}_{1}^{\prime}+\cdots+z_{n-2} \mathrm{e}_{n-2}^{\prime}
$$

with indeterminate coefficients $z_{1}, z_{2}, \cdots, z_{n-2}$. Consider next the equations

$$
f_{\mu, v, \rho, 0, \cdots, 0}\left(\mathfrak{a}_{1}, \mathfrak{a}_{2}, \mathfrak{z}, \mathfrak{o}, \cdots, \mathfrak{o}\right)=0,
$$

where $\mu, \nu, \rho$ range over all systems of non-negative integers with

$$
\mu+\nu+\rho=s, \quad 0<\rho<s .
$$

Again, $\Omega\left(r_{1}^{\prime}, \cdots, r_{h^{\prime}}^{\prime} ; 0\right)$ exists for the degrees $r_{1}^{\prime}, \cdots, r_{h^{\prime}}$ of these equations in $z_{1}, z_{2}, \cdots, z_{n-2}$. It follows for sufficiently large $n$ that the system $(7)$ has a non-trivial solution $\left(z_{1}, \cdots, z_{n-2}\right)$. Let $z=\mathfrak{a}_{3}$ be the corresponding point. Then $\mathfrak{a}_{1}, \mathfrak{a}_{2}, \mathfrak{a}_{3}$ are linearly independent.

Set $t=\Psi(s) .^{4}$ Assuming that $i$ is sufficiently large we continue with our procedure until we obtain $t$ linearly independent points $\mathfrak{a}_{1}, \mathfrak{a}_{2}, \ldots, \mathfrak{a}_{t}$ such that $t^{\mathfrak{b}}$

$$
f_{\mu, \nu}, \cdots, \tau\left(\mathfrak{a}_{1}, \mathfrak{a}_{2}, \cdots, \mathfrak{a}_{t}, \mathfrak{D}, \cdots, \mathfrak{D}\right)=0
$$

for every system of $n$ non-negative indices $(\mu, \nu, \cdots, \tau)$ with $\mu+\nu$ $+\cdots+\tau=s$ in which the first $t$ of our indices are all less than $s$.

For $\mathfrak{x}_{1}=\mathfrak{a}_{1}, \mathfrak{x}_{2}=\mathfrak{a}_{2}, \cdots, \mathfrak{x}_{t}=\mathfrak{a}_{t}, \mathfrak{x}_{t+1}=\mathfrak{o}, \cdots, \mathfrak{x}_{n}=\mathfrak{D}$, the identity

${ }^{3}$ In part 2 of the proof we mean by "sufficiently large $n$ " all values of $n$ which lie above a suitable lower bound $\Lambda(s)$ depending only on $s$.

${ }^{4}$ In the case of Theorem $\mathrm{B}$, we take $t=2$. The equation (8) will have a solution if we extend the field $K$ by the adjunction of an sth root.

5 If one of the last $n-t$ indices in $(\mu, \nu, \cdots, \tau)$ does not vanish, this equation is trivial, since the left side then contains an $\mathfrak{x}_{i}=\mathfrak{o}$ to a positive degree. 
(5) gives a relation

$$
f\left(u_{1} \mathfrak{a}_{1}+u_{2} a_{2}+\cdots+u_{t} a_{t}\right)=\sum_{i=1}^{t} a_{i} u_{i}^{s},
$$

where $a_{i}$ is a certain number of $K$. Actually, $a_{i}=f\left(\mathfrak{a}_{i}\right)$.

Since $t=\Psi(s)$, the equation

$$
\sum_{i=1}^{t} u_{i}^{s} a_{i}=0
$$

has a non-trivial solution $\left(u_{1}, u_{2}, \cdots, u_{t}\right)$ in $K$. The corresponding point $\mathfrak{x}=\sum u_{i} \mathfrak{a}_{i}$ then yields a non-trivial solution of the equation $(\mathfrak{x})=0$ in $K$.

This argument shows the existence of $\Omega(s ; 0)$.

3. We assume that the existence of $m^{\prime}=\Omega(s ; m-1)$ has already been shown. If $n$ is sufficiently large, ${ }^{6}$ the result of 2 shows that we may find a point $\mathfrak{a}_{1} \neq 0$ such that

$$
f\left(a_{1}\right)=0 .
$$

Consider again the equations (6) where $\mathfrak{y}$ has the old significance. Again, $\Omega\left(1,2, \cdots, s-1 ; m^{\prime}-1\right)$ exists. If $n \geqq \Omega(1,2, \cdots, s-1$; $\left.m^{\prime}-1\right)$, it follows that there exists an $\left(m^{\prime}-1\right)$-dimensional linear space $M_{0}$ such that the equations (6) hold for all points $\mathfrak{y}$ of $M_{0}$, and that $M_{0}$ does not contain $\mathfrak{a}_{1}$.

The identity $(5)$ for $\mathfrak{x}_{1}=\mathfrak{a}_{1}, \mathfrak{x}_{2}=\mathfrak{y}, \mathfrak{x}_{3}=\mathfrak{D}, \cdots, \mathfrak{x}_{n}=\mathfrak{o}$ yields

$$
f\left(u_{1} \mathfrak{a}_{1}+u_{2} \mathfrak{y}\right)=u_{2} f(\mathfrak{y}),
$$

on account of (6) and (9). Restricting the point $\mathfrak{y}$ to the linear manifold $M_{0}$, we may consider the coordinates of $y$ as linear homogeneous functions of $m^{\prime}$ parameters $z_{1}, z_{2}, \cdots, z_{m^{\prime}}$. Since $m^{\prime}=\Omega(s ; m-1)$, it follows that there exists an $(m-1)$-dimensional linear subspace $M_{1}$ of $M_{0}$ such that $f(\mathfrak{y})=0$ for all points $\mathfrak{y}$ of $M_{1}$. But (10) shows that $\mathfrak{a}_{1}$ and $M_{1}$ together span an $m$-dimensional linear space $M$ which consists entirely of solutions of $f(\mathfrak{x})=0$. This proves the existence of $\Omega(s ; m)$ which contradicts the assumptions made above.

This finishes the proof of Theorem $C$. The same method yields the proof of Theorem B, and hence the Theorem A.

3. Applications. ${ }^{7}$ Consider the general algebraic equation of degree

\footnotetext{
${ }^{6}$ In part 3 of the proof we shall say that $n$ is sufficiently large if it lies above a suitable lower bound $M(s, m)$, depending on $s$ and $m$ only.

${ }^{7}$ For Hilbert's resolvent problem, see the paper by Segre quoted in footnote 1 and the literature mentioned in this paper, also A. Wiman, Nova Acta Uppsala (1927).
} 
$n$ in one unknown

$$
f(x)=x^{n}+a_{1} x^{n-1}+\cdots+a_{n}=0 .
$$

If the roots are $\omega_{1}, \omega_{2}, \cdots, \omega_{n}$ and if we set

$$
\theta_{i}=u_{0}+u_{1} \omega_{i}+\cdots+u_{n-1} \omega_{i}^{n-1}
$$

then the $\theta_{i}$ are the roots of an equation

$$
g(x)=x^{n}+b_{1} x^{n-1}+\cdots+b_{n}=0
$$

and it is well known that the coefficient $b_{i}$ of this Tschirnhaus transformation is a homogeneous polynomial $B_{i}\left(u_{0}, u_{1}, \cdots, u_{n-1}\right)$ of degree $i$ in the $u_{0}, u_{1}, \cdots, u_{n-1}$. For a fixed $k$, we determine the quantities $u_{0}, u_{1}, \cdots, u_{n-1}$ as a non-trivial solution of the equations

$$
\begin{gathered}
B_{1}\left(u_{0}, u_{1}, \cdots, u_{n-1}\right)=0, \\
B_{2}\left(u_{0}, u_{1}, \cdots, u_{n-1}\right)=0, \cdots, B_{k}\left(u_{1}, u_{2}, \cdots, u_{n-1}\right)=0 .
\end{gathered}
$$

It follows from Theorem $\mathrm{A}$ that for sufficiently large $n$ it is possible to take $u_{0}, u_{1}, \cdots, u_{n-1}$ in a field obtained from the field of the rational functions of $a_{1}, a_{2}, \cdots, a_{n}$ by adjunction of a finite number of radicals. The equation $g(x)$ then has the form

$$
x^{n}+b_{k+1} x^{n-k-1}+\cdots+b_{n}=0 .
$$

Its roots then may be considered as algebraic functions of $n-k$ quantities $b_{k+1}, b_{k+2}, \cdots, b_{n}$. Since $\omega_{i}$ can be expressed in terms of $\theta_{i}$, it follows that the solution of the general equation of $n$th degree can be expressed in terms of the coefficients if we use radicals and one algebraic function of $n-k$ arguments. ${ }^{8}$ Here $k$ was a fixed number and $n$ was to be taken sufficiently large.

Hilbert's resolvent problem deals with the question of finding the smallest number $l_{n}$ for given $n$ such that the roots of the general equation of degree $n$ may be expressed in terms of the coefficients by means of algebraic functions of at most $l_{n}$ parameters. Our above remark shows that $l_{n} \leqq n-k$ for fixed $k$ and sufficiently large $n$. In other words, we have shown that ${ }^{9}$

${ }^{8}$ Since we can make $b_{n}=1$ through a simple transformation, we could replace the last function by one depending on $n-k-1$ arguments.

${ }^{9}$ This result shows that in Segre's notation an infinite series of theorems $\mathrm{H}_{i}$ exists. The same is true for the theorems $B_{i}$, if in the statement beside the adjunction of square roots and cube roots the adjunction of a finite number of other radicals is admitted. On the other hand, icosahedral irrationalities are superfluous. The existence of these infinite series of theorems $\mathrm{H}_{\boldsymbol{i}}$ and $\mathrm{B}_{\boldsymbol{i}}$ had been stated as a conjecture in Segre's paper. 


$$
\lim _{n \rightarrow \infty}\left(n-l_{n}\right)=\infty .
$$

Hilbert's observation that $l_{n} \leqq n-5$, at least for $n \geqq 9$, and Segre's observation that $l_{n} \leqq n-6$, at least for $n \geqq 157,{ }^{10}$ can be supplemented by an infinite number of analogous observations. The method of $\$ 2$ would allow us to find explicit values $n_{k}$ such that $l_{n} \leqq n-k$ for $n \geqq n_{k}$. However, the values obtained would probably be far too large.

As an example of a field which satisfies the assumption $\left(^{*}\right)$ of Theorem C, we may take any field $K$ which is closed with regard to forming radicals $a^{1 / m}, a$ in $K, m=2,3,4, \cdots$. We have here $\Psi(r)=2$ for all $r$. In particular, any homogeneous equation $f\left(x_{1}, x_{2}, \cdots, x_{n}\right)=0$ of degree $r$ has a non-trivial solution, provided that $n$ lies above a certain number depending on $r$ only.

An example of a somewhat less trivial nature is obtained by considering a $p$-adic field $K$. As is well known the multiplicative group of all $\alpha^{r}(\alpha \neq 0, \alpha$ in $K)$ is of finite index in the group of all $\alpha(\alpha \neq 0$, $\alpha$ in $K$ ). From this it follows at once that the assumption $(*)$ of Theorem $\mathrm{C}$ is satisfied, and the statement of Theorem $\mathrm{C}$ holds for $K$. In particular, a homogeneous equation $f\left(x_{1}, \cdots, x_{n}\right)=0$ of degree $r$ in a $p$-adic field has a non-trivial solution $\left(x_{1}, x_{2}, \cdots, x_{n}\right)$, if $n$ is sufficiently large, say $n \geqq N(r) .{ }^{11}$

\section{UNIVERSITY OF TORONTO}

${ }^{10}$ The somewhat rough method of our proof does not allow us to derive this result. The bound obtained for $n$ would be much larger.

$11 \mathrm{E}$. Artin has remarked that it follows at once from the existence of normal division algebras of rank $r^{2}$ over $K$ that $N(r)>r^{2}$. 